\title{
Adolescentes y jóvenes en el entorno digital: análisis de su discurso sobre usos, percepción de riesgo y mecanismos de protección ${ }^{1}$
}

\section{Adolescents and youth in Internet: discourse analysis of uses, risks and protection}

\author{
Belinda de Frutos Torres. Universidad de Valladolid \\ Tamara Vázquez Barrio. Universidad CEU San Pablo
}

Recibido: 9-IX-2011 - Aceptado: 21-VII-2012

Resumen:

El acceso a Internet de los niños y adolescentes es una realidad. El objetivo de este trabajo es conocer la experiencia de uso de Internet en el colectivo de adolescentes y jóvenes y su percepción de riesgo en la Red. La metodología utilizada han sido seis grupos de discusión y una encuesta realizada a 323 jóvenes. Los resultados describen el amplio abanico de actividades que realizan en el medio interactivo y el tiempo dedicado a las mismas en su vida cotidiana. Internet no solo ocupa una parte importante de su tiempo de ocio, sino que además está definiendo sus formas de entretenimiento y su relación con el entorno. En cierta medida son conscientes de los riesgos asociados a la Red, especialmente los relacionados con el control de su privacidad y tienden hacia un uso más racional del medio a medida que es mayor su experiencia en el mismo o van adquiriendo mayor madurez personal.

Palabras clave:

Internet, jóvenes, adolescentes, redes sociales, usos y privacidad.

Abstract:

Internet access for children and adolescents is an upcoming reality. The aim of this paper is to analyze the uses of the interactive media in the group of teenagers and know how they perceive their risk on the Internet. The methodology used has been six focus groups and a survey of 323 young people. The results describe the broad scope of activities they are implementing the media and the time spent in their daily lives. Internet not only plays an important part of their leisure time, but is also defining its forms of entertainment and its relationship with the environment. To some extent they are aware of the risks associated with Internet, especially those related to the control of their privacy and tend towards a more rational use of the medium as it is more experience in the same or are acquiring greater personal maturity.

Keywords:

Internet, young, teenagers, social media, practice and privacy

1 Nota de las autoras: Esta investigación ha sido realizada en el proyecto de investigación con referencia USP-PI-5/09 financiado por la Universidad CEU San Pablo. Las autoras quieren agradecer la colaboración desinteresada de Nielsen/ Net-Rating Spain y Ad-Hoc Investigación de Mercados en el desarrollo de la investigación y por los datos aportados para su realización 


\section{Introducción}

El panorama de consumo de medios ha cambiado desde la generalización de Internet. A lo largo de los últimos años hemos visto como el medio interactivo ha incrementado su penetración progresivamente. En el caso de los jóvenes este cambio ha sido más evidente, constituyen el segmento donde Internet tiene mayor alcance e intensidad de uso. Según los últimos datos del Estudio sobre Equipamiento en el hogar (INE, 2010), el 91,5\% de los niños entre 16 y 25 años acceden a Internet al menos una vez a la semana. Según el último informe publicado por la ONSTI sobre la sociedad en Red, el 61\% de los hogares tiene conexión a Internet y el $62 \%$ de los Españoles se conecta habitualmente (al menos un día a la semana). Tomando como referencia en el día de ayer, este porcentaje es del 44,7\% según la última ola del EGM (AIMC, 2012). En el segmento de los más jóvenes (entre 14 y 19 años) el porcentaje de conexión en el día de ayer se eleva hasta el 71,4\%.

La proliferación de contenidos digitales y el uso activo de las redes sociales ha dado mayor impulso al medio interactivo especialmente entre los más jóvenes. Aunque las cifras oficiales del Eurobarómetro sitúan el uso de las redes sociales en el 35\% de la población, otras fuentes apuntan un alcance bastante más alto. Según la tercera Ola del Observatorio de redes sociales realizado por la consultora The Cocktail Analysis, (2012) la proporción de usuarios que tiene abierto un perfil en las redes sociales se cifra en un $91 \%$ de los internautas. Según estos analistas, se está produciendo la consolidación y la definición del uso de las redes sociales, después de la novedad y el deseo de experimentar y conocer el potencial de esta herramienta, que vivió su máximo crecimiento en los años 2008 y 2009.

Sin duda el binomio Internet y adolescentes es un claro ejemplo de rápida adopción de una innovación con implicaciones que trascienden el hábito de consumo de medios. Como apunta la investigadora Rubio (2008: 79): "Internet es un fenómeno social total, no se trata exclusivamente de un medio de comunicación, ni de un medio de información, sino que se manifiesta en una relación social plena, internacional, supraindividual y suprafuncional". La revolución que ha supuesto la red en diversas esferas de ámbito cultural, social y económico, ha calado de forma extraordinaria en los jóvenes y adolescentes que han visto un medio amigo en el que canalizar sus intereses al margen de la cultura dominante. Internet constituye una gran ventana al mundo con un sinfín de oportunidades nuevas de socialización.

El colectivo de los más jóvenes ha adoptado de forma rápida y positiva las aplicaciones y posibilidades que ofrece este complejo entorno de forma natural. A ello ha contribuido, entre otros aspectos, la sencillez de uso, puesto que las aplicaciones están orientadas al uso intuitivo y no requieren destrezas o habilidades desde el punto de vista tecnológico; la facilidad de acceso con la implantación de la banda ancha incorporada en los hogares y la valoración positiva del medio desde el entorno educativo y en el familiar. Internet no solo se consume, sino que se comparte. A diferencia de otros medios de comunicación de masas, constituye un espacio en el que desarrollarse personalmente, quizá ahí resida la clave de su éxito. 
Los jóvenes y adolescentes utilizan Internet principalmente para comunicarse, entretenerse y para buscar información. Las diferencias en función de la edad respecto a esta triple utilización son bastante exiguas entre estos dos grupos, jóvenes y adolescentes, pero sí son importantes respecto al colectivo más joven, los niños. Según la última encuesta de Equipamiento del Hogar del INE, el uso de tecnologías de información es, en general, muy elevado entre la población infantil (10-15 años). El uso de ordenador es prácticamente universal (94,6\%) y el 87,3\% utiliza Internet, dato que alcanza el 92\% para los niños entre 14 y 15 años. La Encuesta Generaciones Interactivas en España indica que el uso de Internet y, sobre todo, del ordenador, es una práctica mayoritaria también en los niños menores de 10 años. El 95\% de los niños de 6 a 9 disponen de PC y el 71\% de conexión a Internet. En el estudio realizado por la Red Eukids on line la edad media de uso de internet por primera vez es a los 9 años (Garmendia, Garitaonandia, Martínez y Casado, 2011). Los datos demuestran que el uso de Internet está, por lo tanto, muy generalizado, pero también indican que la utilización que se hace de la Red varía en función de la edad. Según un estudio del Defensor del Menor, los niños más pequeños utilizan Internet fundamentalmente para buscar información (39'5\%), para jugar (28'5\%), para chatear (20’5\%) y para buscar música (11'5\%). La utilización de las redes sociales empieza a los 11-12 años y se generaliza a los 14. A partir de esta edad el uso de redes sociales supera el $80 \%$ hasta alcanzar una cota máxima de uso del 85\% a los 17 años. Según el estudio Generación Nacido Digital de Conecta, el porcentaje de adolescentes que dice haberse registrado por primera vez en una red social con menos de 14 años es de un $10 \%$.

A medida que van creciendo el uso que hacen de Internet cambia y lo que buscan principalmente cuando navegan es relacionarse y ahí es donde adquieren gran importancia las redes sociales. Según un estudio de la Fundación Pfizer (2009) diseñado conjuntamente con TNS Demoscopia, casi la mitad de los jóvenes, el 48,8\%, participa o accede a las mismas diariamente, y cerca de 8 de cada 10 (77,6\%) lo hace al menos dos o tres veces a la semana. Un porcentaje algo superior refieren Garitaonandia y col. (2011) con un 56\% de menores con perfil en redes sociales. Este porcentaje es mayor a medida que avanzamos en el grupo de edad, situándose en el 89\% entre los niños de 15 a 16 años. El índice de uso frecuente de las redes sociales (todos o casi todos los días) correlaciona con la edad. Los que menos se conectan son los jóvenes de 11 a 13 años $(29,9 \%)$ y los que más los de 17 a 20 (58,3\%), pero las diferencias son todavía más significativas en cuanto a los otros dos usos: el entretenimiento y la búsqueda de información según pone de manifiesto el estudio de la Fundación Pfizer.

Las implicaciones de la rápida adopción de Internet por el público infantil y juvenil es objeto de interés para instituciones, educadores e investigadores, además de los estudios sectoriales o que sirven a intereses económicos (Sánchez y Frutos, 2010). Una de las líneas de trabajo más interesantes y fructíferas se desarrolla desde el proyecto EU Kids on line sobre los riesgos asociados al consumo. Desde este trabajo se apunta que se tiende a sobrestimar las habilidades de los jóvenes en la red y subestimar su necesidad de apoyo y guía (Livingstone, 2010). Según Garitaonandia y Garmendia (2009), en España los padres se muestran más preocupados que la media europea por el control de lo que hacen sus hijos en la red, pero hay un bajo nivel de mediación parental comparado con el resto de Europa. Un 66\% no controla la navegación de sus hijos 
por Internet, cuestión que se ve confirmada en el estudio de Brigué y Sádaba (2009; 2011), en el que constatan que el 85,5\% de los niños navegan solos y que el 70,9\% afirma haber aprendido sin ningún tipo de ayuda a navegar. A datos similares llegan Arrizabalaga, Aierbe y Medrano (2010) en un estudio publicado más recientemente.

El trabajo publicado por Hasebrink, Livingstone y Haddon (2008) a nivel europeo apunta que cuanto más tiempo están los niños en el medio interactivo y cuanto más usan determinados servicios, más se incrementa la probabilidad de encontrar experiencias positivas y negativas. Al margen de las preferencias de los niños por comportamientos que acarrean mayor o menor riesgo, las habilidades de los niños en la red y la educación en el medio puede, en buena medida, aliviar los riesgos. La mediación de los padres es crucial para minimizar los riesgos asociados al uso, según apunta la investigadora Sonia Livingstone (2009). Entre los riesgos asociados al medio interactivo está la adopción de patrones de referencia que se alejan de los valores educativos (Elzo, 2009). Por otra parte, los jóvenes son bastante escépticos respecto a los mensajes publicitarios en la red, sin embargo se muestran bastante más receptivos en las acciones y eventos que las marcas proponen en la misma (Frutos y Sánchez, 2011).

El objetivo de este trabajo es indagar en cómo se ha integrado el consumo de los medios interactivos en los jóvenes y adolescentes. En particular se analiza, por un lado, el uso y consumo que hacen de la Red y, por otro, la percepción de riesgo en el medio interactivo y los mecanismos de protección. Así mismo se explora la evolución que se produce en el discurso de los jóvenes a medida que el colectivo adquiere independencia y autonomía en el uso del medio con el paso de la edad.

\section{Metodología}

El estudio se ha realizado combinando la metodología cualitativa y cuantitativa. Para el estudio cualitativo se ha empleado la técnica de los grupos de discusión. Se consideró que esta técnica aporta una aproximación más cercana y real de la experiencia de los menores en Internet. Se valoró positivamente que el hecho de compartir sus experiencias con el resto de los miembros del grupo producía un efecto facilitador, y por ello, se conseguiría un discurso más rico. Por otra parte, los grupos de discusión sirvieron para el desarrollo del cuestionario. Se identificaron las actividades más relevantes dentro de su experiencia, lo que permitió sistematizar y cuantificar con mayor exactitud las respuestas al mismo. La combinación de ambas metodologías permite un enriquecimiento mutuo, puesto que el análisis del discurso proporciona un acercamiento a la experiencia de uso, mientras que el cuestionario facilita conocer su alcance.

El trabajo se ha realizado con jóvenes de edades comprendidas entre los 15 y los 19 años. Los participantes fueron elegidos al azar entre los alumnos de clase correspondientes a $4^{\circ}$ curso de ESO, 1 y $2^{\circ}$ de Bachillerato y primer curso de Universidad de dos centros educativos ubicados en Madrid y en Segovia. En total se llevaron a cabo 6 sesiones de discusión, tres con grupos del perfil de jóvenes en su primer año en la universidad y otros tres grupos de adolescentes. La duración de las sesiones fue aproximadamente de 60 minutos. 
El estudio cuantitativo se realizó mediante una encuesta que se aplicó a 323 alumnos procedentes de 6 centros de enseñanzas medias ubicados en Madrid, Segovia, Ciudad Real y Málaga, de carácter público, privado y concertado. El rango de edad de los participantes se situó entre los 14 y 19 años, siendo la edad media de 16,3 años. El 37,8\% son hombres y el $62,2 \%$ son mujeres. Las sesiones de los grupos de discusión y la encuesta se llevaron a cabo entre los meses de abril y mayo de 2011.

\section{Resultados del análisis}

\subsection{Uso y consumo de Internet}

El discurso sobre el uso de Internet gira en torno a tres grandes ejes: la comunicación, el entretenimiento y la información, que difieren en importancia y dedicación. Si hubiera que priorizar entre los tres, Internet es un medio ante todo de comunicación. Los datos de la encuesta nos permiten cuantificar la intensidad y el perfil de consumo. El 87,3\% declara que accede a Internet todos los días, el 12,4\% se conecta de vez en cuando y solo uno de los participantes afirma que nunca lo utiliza fuera del centro de estudios. La proporción de conexión diaria es semejante entre los 3 niveles educativos, según se ha comprobado mediante un análisis de contingencia (chi-cuadrado=2,978, gl=4, sig.=0,561).

El tiempo dedicado al medio interactivo es bastante alto, la mitad de los encuestados permanecen conectados 5 horas y 48 minutos $^{2}$ al día durante la semana y durante el fin de semana el consumo aumenta alcanzando las 8 horas y 18 minutos. La incorporación de los dispositivos smartphone, que permiten estar conectado las 24 horas del día, sin duda contribuye al incremento del consumo, según se puso de manifiesto en los grupos de discusión ${ }^{3}$. No obstante hay una clara diferencia en el consumo, los usuarios más moderados, aproximadamente el $24 \%$ de los encuestados, permanecen en la red menos de dos horas a diario y durante el fin de semana menos de 3 horas y media. En el otro extremo, los usuarios con un perfil más intenso, se conectan más de 7 horas al día durante la semana y por encima de las 10 horas y media en el fin de semana.

Ante la posibilidad de que existan diferencias en el consumo asociadas a la edad, se ha comparado el tiempo de conexión entre los tres cursos académico ( $4^{\circ} \mathrm{ESO}, 1^{\circ} \mathrm{y} 2^{\circ}$ de Bachillerato) mediante un ANOVA intersujetos. Los resultados muestran que hay diferencias estadísticamente significativas en el consumo semanal y de fin de semana asociadas al curso

2 La dispersión en el tiempo dedicado al medio influye en el cálculo de la media aritmética, tomando como referencia la mediana se puede afirmar que la mitad de los jóvenes están conectados durante 3 horas y 18 minutos a diario y durante el fin de semana el valor alcanza las 6 horas y media.

3 Los datos de la encuesta indican que el 4,4\% está conectado a Internet durante todo el día durante la semana y el 7,9\% durante el fin de semana. 
académico ( $\mathrm{F}=6,011$; sig. =,003; eta=,037 y F=5,265; sig. =,006; eta=,033). Al analizar la tendencia se aprecia que en el curso superior ( $2^{\circ}$ de Bachillerato) el consumo es más moderado, obtiene una media de 4,2 horas a diario y 6,8 horas durante el fin de semana; en contraste el curso correspondiente a $1^{\circ}$ de Bachillerato el consumo es significativamente superior a los otros dos grupos, con una media diaria de 7,8 horas y 10,8 durante el fin de semana.

Figura 1. Tiempo de consumo en Internet por nivel educativo

\section{Tiempo de consumo de Internet} (Media aritmética)

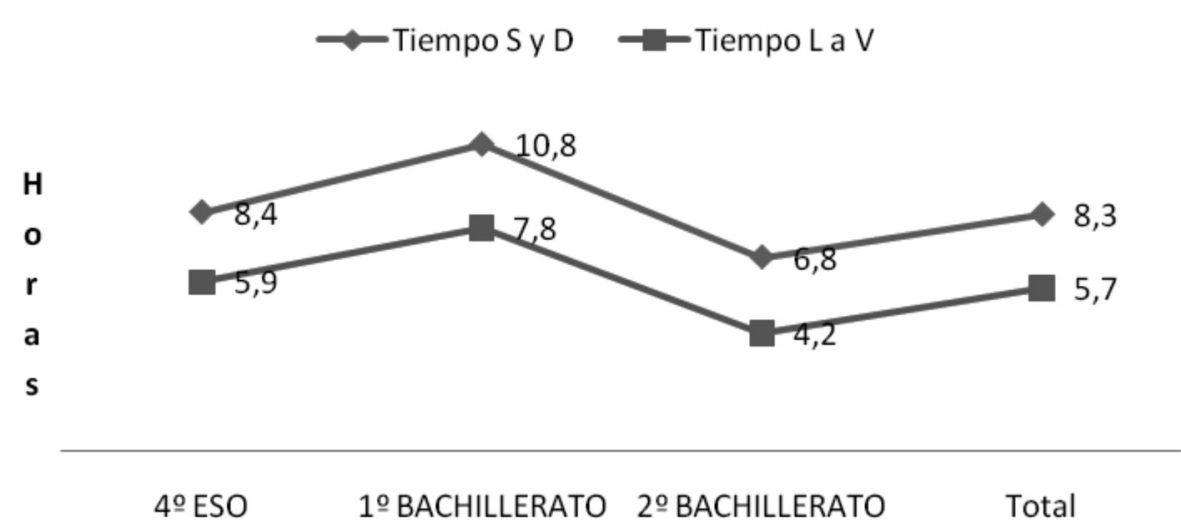

\subsubsection{Internet y el entretenimiento}

Los contenidos audiovisuales: películas y series

El consumo de contenidos audiovisuales está presente en todo el colectivo, si bien se observan dos formas diferenciadas de recepción: bajar los contenidos (descargas) o verlos directamente en streaming. El visionado en directo evita los extensos tiempos de espera en las descargas, tarea que puede llevar varios días y no obliga a tener un lugar de almacenamiento de alta capacidad. La principal desventaja es que los sitios que ofrecen el visionado de forma gratuita solo permiten ver $72 \mathrm{mi}-$ nutos seguidos, pero lejos de ser un obstáculo encuentran fácilmente una forma de solucionar el problema, como manifiesta una de las participantes: "lo apagas y vuelves aunque tienes que buscar otra vez lo que estabas viendo" (Grupo 2, jóvenes). Para los que optan por grabar los contenidos consideran valioso decidir el momento de consumo. Los posibles inconvenientes asociados al tiempo de descarga, se evitan bajándose los contenidos en bloques, de modo que el visionado se produce, al cabo de los días, cuando tienen varios capítulos de una serie o varias películas. 
"Me gusta descargar las películas, tenerlas en el ordenador y cuando me apetece las veo. Descargarlas y luego verlas enteras, a lo mejor tengo 15 (películas), descargadas en una semana o el tiempo que sea, cuando las tengo enteras las veo y mientras tanto me estoy descargando otras 15” (Grupo 1, jóvenes). "(En streaming) si la velocidad de descarga no es buena se puede ver interrumpida o hay que esperar" (Grupo 2, jóvenes).

Ambos, jóvenes y adolescentes, participan de la descarga de contenidos, si bien los adolescentes manifiestan en los grupos de discusión preferencia por verlos directamente en streaming y solo se los bajan si es por encargo para el consumo de terceros, por ejemplo, los padres o abuelos. A pesar de bajar pocos contenidos reconocen haberse llevado alguna sorpresa cuando creían estar bajándose una película y en el momento del visionado descubrían que era otra, generalmente con contenidos sexuales: “te puede aparecer alguna cosa... no te dejan ver lo que es. Tienes que descargarlo y luego lo puedes ver" (Grupo 3, adolescentes).

Aunque los adolescentes todavía siguen los contenidos audiovisuales principalmente a través de la televisión, coinciden con los jóvenes en que la principal ventaja de ver los contenidos de ficción en la red es la posibilidad de elegir el momento de recepción. Además Internet ofrece mayor abanico de títulos, tanto en las películas como en las series, en las que, por otro lado, hay disponible cualquier temporada ordenada cronológicamente, de modo que pueden decidir exactamente qué capítulo visionar. Este hecho cobra mayor relevancia en las series extranjeras, puesto que pueden acceder a los capítulos que aún no están pasando por televisión. Citan la serie de suspense Lost, haciendo referencia a que en lugar de esperar una semana para ver el desenlace, pueden ver el siguiente episodio de forma inmediata. El visionado a través de la red permite seguir la secuencia de las temporadas y ver todos los capítulos, que no siempre se respeta en las cadenas de televisión. Por otra parte, series de gran éxito en televisión tienen sus fans en Internet que siguen su propia cronología de visionado. Comienzan por la primera temporada para poder seguir la trama cronológicamente.

.. es lo bueno de estos sitios, que vienen todos los capítulos por temporada, y te permite ver un capítulo, sabes cuál te falta por ver o incluso series que te han gustado cuando eras pequeña, pues la puedes volver a ver (Grupo 2, jóvenes).

"Lo malo es que lo suelen echar un lunes por la noche y hasta el lunes siguiente no lo vuelves a ver, al ritmo que ellos dicen, a la hora que ellos dicen, imagina que ese día tienes un trabajo o lo que sea, te da mucha comodidad" (Grupo 1, jóvenes).

Para las series que están en la parrilla de televisión, la ventaja de Internet es que no hay publicidad, eso les permite ver el capítulo seguido, sin los veinte minutos de pausas publicitarias. La posibilidad de escuchar la versión original subtitulada es otro plus que se menciona para las series extranjeras.

Los sitios web de visionado de películas (megaupload, seriesyonkies, etc.) se convierten en fuente de referencias para conocer las novedades, tanto en películas como en las series. Los comentarios de otros usuarios son testimonios útiles para 
elegir las series, pero verdaderamente son los amigos los que influyen más, recomiendan una serie y después de ver un capítulo deciden si lo siguen o no. Ante este abanico de posibilidades, con una programación mucho más amplia de la que ofrece la parrilla de televisión, cambia el patrón de visionado, no se siguen muchas series en Internet al mismo tiempo, mencionan dos o tres, pero estas se consumen en dosis más altas (varios capítulos cada vez, de diez en diez); además hay que unir las series que emiten por televisión, de las que cada día de la semana tienen una favorita. El consumo de la ficción en Internet es claramente individual.

Figura 2. Actividades que realiza en la red en porcentaje

\section{Actividades que realiza en la red}

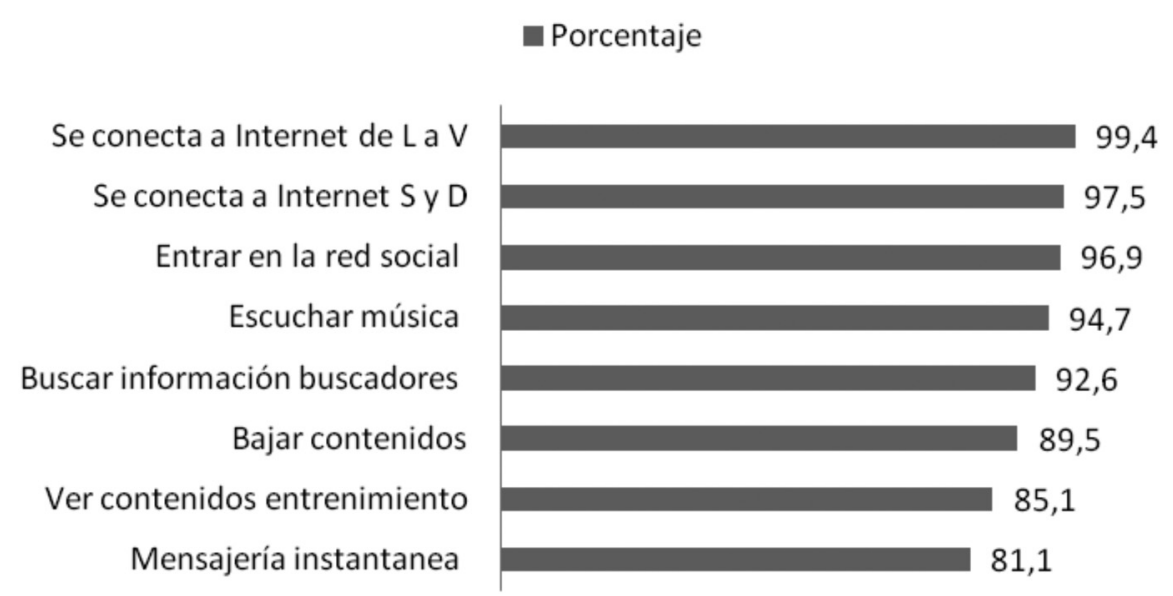

El hecho de no residir en domicilio familiar ha hecho que los grupos de estudiantes de primer curso de universidad se hayan visto forzados al cambio y han sustituido el tiempo que dedicaban al televisor por Internet. En cambio, los adolescentes siguen viendo sus series preferidas en el televisor, salvo que por algún motivo no puedan seguir la programación.

“con la falta de televisión... en casa no veía mucha TV, pero ahora he sustituido lo que veía en televisión por Internet" (Grupo 2, jóvenes).

Los datos de la encuesta corroboran lo extendido de ambas prácticas. El 89,5\% afirma que alguna vez se ha bajado contenidos de entretenimiento de la red para archivarlo y verlo en otro momento. El seguimiento es similar para el conjunto de jóvenes, puesto que no se han encontrado diferencias estadísticamente significativas al compararlas por la edad (Chi-cuadrado = 1,479 g.l.=2, sig.=0,477). Al cuantificar el tiempo que le dedican a lo largo de un día, los jóvenes emplean por 
término medio 57 minutos diarios ${ }^{4}$. Por otra parte, el 85,1\% ve contenidos de entretenimiento directamente en Internet, en este caso se constatan diferencias significativas al compararlo entre los tres grupos de edad (Chi-cuadrado=8,8 g.l. $=2$ sig.=0,012). Los adolescentes afirman con mayor frecuencia seguir contenidos de ficción a través de Internet $(95,6 \%)$, a pesar de que en los grupos de discusión reconocían que siguen viendo sus series favoritas en televisión. El tiempo que dedican a esta actividad por término medio es de una hora y 22 minutos 5 .

La música y los videojuegos

La música es un elemento importante en las vidas de estos jóvenes y adolescentes e Internet es el medio preferido de recepción, de hecho es la actividad de entretenimiento más generalizada con un seguimiento del 94,7\%, sin que haya diferencias significativas atribuibles a la edad (Chi-cuadrado=0,141 g.l. $=2$ sig.=0,932). Al igual que con la recepción de los contenidos audiovisuales coexisten dos patrones de consumo: bajar los contenidos o la recepción en tiempo real. El afán por bajar contenidos ha ido decayendo con el paso del tiempo y en general prevalece la idea de que cualquier contenido está siempre disponible en la red. Tener sus propias listas de reproducción, o la posibilidad de pasar los contenidos musicales a otros dispositivos (a través del mp3) son las razones por las que se justifican las descargas. Así lo manifiesta una de las participantes: “la música me la descargo y la paso al MP4" (Grupo 3, jóvenes). A su vez, la recepción desde la red toma diferentes modalidades, escuchar la radio a través de Internet, al mismo tiempo que se llevan a cabo otras actividades, o bien desde sitios web especializados en contenidos musicales como Spotify. También es relevante ver vídeos musicales en Youtube, que ofrece una forma rápida de llegar a los grupos que les gustan y además, permite explorar nuevos temas, canciones relacionadas, etc. El tiempo dedicado a escuchar música es de una hora y 15 minutos ${ }^{6}$ en promedio.

El tercer aspecto relacionado con el entretenimiento lo ofrecen los videojuegos en la red. En el momento que emerge este aspecto en el discurso todos los participantes reconocen que han tenido alguna experiencia con los videojuegos. No se identifican como jugadores intensos (adictos al videojuego), de hecho esta actividad, por alcance (62,8\%) y dedicación (media de 50 minutos $^{7}$ ) es la que tiene menos éxito en el colectivo, no obstante coinciden en afirmar que han pasado alguna etapa enganchados a algún videojuego.

Los participantes en los grupos de discusión encajan más en un perfil de usuario ocasional, que en un momento determinado explora un juego y juega con cierta intensidad, pero que con el tiempo decae su interés. Las experiencias de los

4 Tomando como referencia la mediana se puede afirmar que la mitad de los jóvenes baja contenidos durante 20 minutos diarios, y tres de cada cuatro no le dedican más de 30 minutos.

5 Tomando como referencia la mediana se puede afirmar que la mitad de los jóvenes consume contenidos audiovisuales durante 60 minutos diarios y el $25 \%$ de los jóvenes le dedican más de dos horas.

6 Tomando como referencia la mediana se puede afirmar que la mitad de los jóvenes escucha durante 50 minutos diarios.

7 Tomando como referencia la mediana se puede afirmar que la mitad de los jóvenes juega durante 30 minutos diarios y el $75 \%$ no supera los 38 minutos. 
juegos on-line sigue este patrón. Conscientes de los peligros asociados a los videojuegos por su capacidad absorbente suelen evitar entrar en ellos, pero se encuentran diferencias significativas asociadas a la edad, los chavales entre 14 y 15 años son los que juegan con más frecuencia (Chi-cuadrado=6,163 g.l.=2 sig.=0,046).

“...me han dicho, no te metas en juegos que luego te picas mucho (Grupo 1, jóvenes)". “Cuando estoy aburrida, el último recurso es un juego de cocina que es muy estresante (Grupo 2, jóvenes)".

\subsubsection{Búsqueda de información referencial}

Internet es una fuente de información sin límites de espacio que ofrece el uso más intelectual del medio. El uso de Internet como fuente de documentación contrastada está asociado a las actividades relacionadas con los estudios, desde donde se incentiva su uso. La wikipedia y el wordreference se han convertido en sitios de referencia para los jóvenes, al igual que otras páginas, como el rincón del vago. Nos ha sorprendido que los adolescentes son muy conscientes de la falta de fiabilidad de la información que ofrecen estas páginas y que, aunque las utilizan, buscan la forma de comprobar la validez o veracidad de los contenidos.

“yo cuando busco algo en Internet para fines académicos, no me meto en una página, no me meto en Wikipedia, me meto en páginas rebuscadas y siempre que vea que toda la información es la misma en todas las páginas. Eso siempre lo hago" (Grupo 4, adolescentes)..

Mientras que para los adolescentes la búsqueda de información se limita a la que necesitan para "las cosas del colegio" (Grupo 4, adolescentes) y para estar al día sobre cosas que les gustan (música, decoración, moda, etc.), los jóvenes acceden en la red a software, programas, actualizaciones y aplicaciones que responden a otro tipo de contenidos. Estas acciones se llevan a cabo con cierta regularidad, si bien no acaparan mucho tiempo en las sesiones de navegación. La función informativa de los medios de comunicación convencionales también tiene su espacio en la red. Los universitarios siguen la información de actualidad a través de las páginas webs de periódicos, revistas, y otro tipo de publicaciones, e incluso los tradicionales canales de televisión se convierten en fuentes de referencia sobre la actualidad relacionada con el medio, los famosos, y otros referentes del colectivo. Este tipo de información, sobre todo la de actualidad, no es buscada habitualmente por los adolescentes.

Los datos de la encuesta confirman lo generalizado que está este hábito, corroborado por el 93,3\% de los encuestados sin que haya diferencias de edad (Chi-cuadrado=1,169 g.l.=2 sig.=0,558). Por término medio se dedica 40,5 minutos al día ${ }^{8}$. Leer noticias y otro tipo de contenidos en páginas de actualidad, es algo menos frecuente que se confirma en el 66,1\% de los casos independientemente de la edad (Chi-cuadrado=4,156 g.l.=2 sig.=0,125). El tiempo dedicado a esta actividad es de 23 minutos ${ }^{9}$.

8 Tomando como referencia la mediana se puede afirmar que la mitad de los jóvenes dedica 20 minutos a buscar información, y la mayor parte dedica entre 10 y 30 minutos (centil 25 y Centil 75).

9 Tomando como referencia la mediana la mitad de los jóvenes dedica 15 minutos a leer contenidos de actualidad. 


\subsubsection{La publicidad gráfica integrada}

La faceta consumidora tiene su reflejo en la actividad en la red. La red ofrece amplia información relevante para la toma de decisiones de compra. El colectivo se deja seducir y vive la experiencia de la búsqueda de información sobre productos o servicios como una faceta más del entretenimiento que ofrece la red. La búsqueda se dirige tanto a las fuentes oficiales (anunciantes) como a los comentarios de otros usuarios que dejan en los foros de compra venta o relacionados con los productos. Explorar la oferta de productos o servicios se lleva a cabo, incluso aunque no se haya activado el proceso de toma de decisiones de compra. Una de las participantes afirmaba: "me encanta mirar vuelos baratos por el placer de saber que están ahí, aunque no tengo previsto irme a ningún sitio" (Grupo 1, jóvenes).

Mientras que los jóvenes y también los adolescentes reconocen que sus procesos de decisión de compra se ven afectados por las opiniones de otros usuarios, todos coinciden en que evitan y no hacen caso a los contenidos publicitarios.

La presencia de la publicidad integrada forma parte del entorno en la navegación por la web. La inversión publicitaria en la red se ha duplicado en los últimos cuatro años y se consolida como una de las vías de financiación del medio (Frutos y Valle, 2011). El análisis de los contenidos publicitarios de la páginas web más visitadas por los más jóvenes da idea del tipo de mensajes a los que está expuesto este colectivo. Un estudio exploratorio de las páginas visitadas por adolescentes mostró que existe un predominio de las creatividades dirigidas a un público adulto caracterizadas por un alto contenido de referencias sexuales, al mismo tiempo que en algunos casos se ofrece una información sesgada que puede incitar a engaño (Sánchez y Frutos, 2010). Aspectos que posteriormente se han confirmado en un estudio posterior donde se constata la presencia de mensajes publicitarios que de forma indiscriminada reclaman la atención mediante estrategias poco edificantes en un entorno con escasa regulación sobre sus contenidos y que está presente particularmente en las páginas que ofrecen de forma gratuita el acceso a series, películas y otros (Villalobos y Frutos, en prensa).

La publicidad tradicional (on display) no consigue atraer su atención y tiene una imagen bastante negativa. La presencia de publicidad que utiliza estrategias poco éticas que juegan con la honestidad del mensaje influye enormemente en esa imagen negativa con la que se asocia la publicidad en la Red. Los reclamos tipo: "has ganado un viaje..." “haz clic aquí y te enviamos..." son algunos ejemplos de esta estrategia que les ha generado experiencias desagradables y se muestran bastante escépticos. Bajo esta misma estrategia se ofrecen otro tipo de reclamos como participar en un pequeño juego (responder una pregunta tipo test de inteligencia, adivinación o similares). Este tipo de gancho les ha llevado a participar, pero denuncian la falta de credibilidad de la publicidad, sobre todo la que se esconde detrás de anunciantes como casinos, contactos y similares.

"La publicidad es muy cargante ...cuando estás bajando contenidos, si no tienes la versión Premium te bombardean con publicidad ... sale por todos los lados (Grupo 2, jóvenes)". 
Otra fuente de experiencias que configura la percepción de la publicidad procede de los formatos denominados flotantes, que utilizan la animación e interactividad como recurso creativo. En esta categoría se encuentran los pop-ups, pop-under, layers, etc. Su rechazo procede de la ausencia de control por parte del usuario y el miedo a virus o programas maliciosos que se puedan instalar en el ordenador. Otro tipo de acciones publicitarias como las sobreimpresiones en los vídeos de Youtube, las cortinillas previas a los capítulos de las series o el acceso a una página de actualidad no se califican como molestas.

Tanto adolescentes, como jóvenes conocen otras acciones publicitarias de la red. Mencionan los videos (marketing viral), los concursos y juegos desarrollados por una marca, e incluso los eventos que llegan a través de la red social. Acciones en las que no emerge el componente de rechazo de la publicidad, al contrario, se perciben como ocasiones para el entretenimiento. Uno de los participantes mencionaba algunos ejemplos: "Yo me acuerdo de algunos que están muy bien,... los de mixto y mixta son super graciosos" (Grupo 3, jóvenes)".

En general se muestran reticentes a aceptar invitaciones para participar, especialmente si no identifican quien es la fuente, y paran en el momento en el que les piden registrarse. Mencionan experiencias en las que después de haber dado su cuenta de correo electrónico han recibido un sinfín de e-mails con ofertas que no estaban relacionadas con la temática y les resulta complicado darse de baja en el servicio.

Por ello, la credibilidad de la fuente juega un papel determinante en la recepción del mensaje o de la acción publicitaria. Un aspecto que se ve reforzado por las invitaciones o recomendaciones de sus conocidos. De hecho las acciones y los eventos que llegan a través de la red social tienen mayor receptividad y probablemente tengan mayor poder persuasivo.

\subsubsection{El microbloging a través de las redes sociales}

Las redes sociales son, con diferencia, el aspecto más importante que ofrece Internet a estos jóvenes, una herramienta que hace realidad su necesidad de relación con su grupo de iguales, que les permite mostrarse a los demás, conocer y explorar en un escenario de interconexiones sin limitaciones espacio-temporales, en cualquier momento y desde cualquier sitio.

"Lo más importante de Internet son las redes social (¿por qué?). Es más cómodo, no sé, hablas con la gente; es una forma de relacionarte con todo el mundo, siempre que quieras y con gente que igual no te haces llamarla, o no puedes llamarla. Estar en contacto con amigos o con conocidos, contactos aunque no sean amigos" (Grupo 2, jóvenes)

La red social Tuenti se posiciona como la principal red para el segmento de edad de los 16 a los 25 años (The Cocktail Analysis, 2010). Todos tienen cuenta en esta red y de forma rápida ha recolocado la función de otras herramientas de comunicación instantánea como el mesenger o incluso el teléfono móvil, que en épocas anteriores de su vida habían tenido 
un protagonismo total en la relación con los demás. El efecto de grupo es fundamental para entrar en la red. El hecho de encontrar a tu grupo de amigos en la red social es determinante en la elección. Posiblemente esta razón ayuda a explicar la penetración que tiene Tuenti en España, Red que no está presente en otros países y que entra en competencia con otras con presencia internacional como Facebook.

"antes del Tuenti utilizaba el Mesenger pero no mucho, ahora lo utilizo mucho menos, he empezado fuerte con el Tuenti este curso, además no tienes a tus padres diciendo deja el ordenador..." (Grupo 2, jóvenes). "Yo el Messenger lo dejé de utilizar desde el Tuenti, pero además no me gusta meterme porque además lo que tengo son contactos que no tengo ni idea de quien son, y de hecho si me meto ahora es porque tengo a gente que le va mal el Tuenti y nos comunicamos por el Messenger ..." (Grupo 1, jóvenes).

“yo me lo hice (Tuenti) de los primeros en el año 2007 y fue porque una amiga que estaba en primero de carrera me dijo, oye te voy a invitar a un sitio que es una especie Messenger pero no es directo, puedes subir fotos, ... hasta que no se empezó a meter todo el mundo en el 2008 yo pasaba, alguna foto tenía subida y ahí empecé un poco" (Grupo 1, jóvenes). "Yo utilizo más Tuenti porque es donde están mis amigos. Facebook la utilizo solo para mantener contacto con amigos del extranjero" (Grupo 5, adolescentes).

Las redes sociales han redefinido el uso de otros dispositivos y aplicaciones que con anterioridad dominaban la relación con su entorno social. El móvil ha quedado relegado al plano de lo inmediato "darte un toque cuando has llegado o vas a salir, por si acaso no han leído el Tuenti”. Es más barato mandarte un mensaje privado por el Tuenti que enviar un SMS por el móvil. Por otra parte, el Messenger (de Microsoft) pasa a un segundo plano según declaran los jóvenes. En muy poco tiempo las plataformas de redes sociales han incorporado la posibilidad del chat y por lo tanto no es necesario conectarse desde otra aplicación ${ }^{10}$. En el grupo de jóvenes el Messenger está asociado a su etapa adolescente, con los contactos de otra época. La redefinición también afecta al correo electrónico, que en el grupo de los jóvenes tiene una vertiente funcional, mantener una correspondencia oficial con profesores, enviarse archivos o enviar trabajos. Al mismo tiempo, ha dejado de ser eficaz al recibir una gran cantidad de spam y correo no deseado, alimentado por los sitios en los que es necesario registrarse a través de una cuenta de correo. Una de las participantes lo ilustraba del siguiente modo: "abres el

10 A pesar de que en los grupos de discusión se sugería que las redes sociales habían desplazado al messenger, encontramos que la conexión simultánea a través de mensajes por el ordenador sigue teniendo bastante importancia, no obstante, no se produce necesariamente a través de la aplicación de Microsoft. Según la encuesta el 81,8\% utiliza este tipo de aplicaciones y el tiempo dedicado a lo largo de un día es superior al tiempo dedicado a la conexión en la red social. En promedio se dedican una media de 4 horas al día. Como en todas las medidas de tiempo la variablidad es muy alta, la mitad de los jóvenes se conectan durante 80 minutos o menos (mediana) y un 25\% de los jóvenes emplean más de 4 horas diarias. El análisis del patrón de consumo se lleva a cabo mediante un ANOVA intersujetos para la edad y para el nivel de educación, siendo significativo el efecto del curso ( $\mathrm{F}=4,506$; sig. =,012; eta=,051). Los estudiantes de $1^{\circ}$ de Bachillerato son los que dedican más tiempo a diario con una media de 6 horas y media, los estudiantes de $4^{\circ}$ curso obtienen un consumo algo más moderado con una media de 3 horas y 42 minutos, y de nuevo, el segundo curso de bachillerato es el que tiene un consumo más bajo con una media de dos horas y 48 minutos. 
correo y tienes 1.500 mensajes en la bandeja de entrada sin leer" (Grupo 3, jóvenes). Curiosamente la forma de limpiar este correo basura es abrir una nueva cuenta de correo, a la que ya solo se incorporan los contactos con los que tienen una relación fluida.

Tuenti ha sido la primera experiencia en redes sociales para los adolescentes, pero no para los jóvenes, que recuerdan el uso de otras redes más primitivas como el Fotolog, que permitía colgar fotos y comentarlas, con un primario sistema de red abierta a todos los integrantes de la red. Aunque también contaba con bastantes seguidores, nunca consiguió los niveles de uso actual.

Muchos de ellos también tienen cuenta en Facebook, una red social con la que, a pesar de ofrecer mucha más versatilidad, no se sienten tan implicados (Monge y Olabarría, 2010). Tuenti se asocia con un uso más intuitivo y sencillo de manejar y en el que están masivamente sus amigos y personas más jóvenes. Por otra parte, Facebook se considera una red más formal, para todas las edades e internacional, se mantiene abierta para determinadas personas a las que no permiten el acceso en Tuenti, por ejemplo, residentes en el extranjero o familiares. Pueden tener agregados a sus padres en Facebook pero bajo ningún concepto los agregarían en Tuenti.

"(Tuenti) Lo tienen chicas de 14 años... pero no te encuentras a padres, en cambio en Facebook tienes personas de todas las edades". "Si veo a mi padre en Tuenti digo qué hace aquí, en cambio Facebook tienes personas de 20, de 30 o de 40 hay profesores, hay padres" (Grupo 2, jóvenes).

Uso de la red social

El 96,9\% declara que habitualmente entra en la red social en las sesiones de navegación, la actividad en la que coinciden mayor número de jóvenes, no obstante en la comparación entre los grupos de edad se constata que entre los de mayor edad, 18 y 19 años, el porcentaje de seguidores habituales de la red desciende ligeramente hasta el 90,9\% (Chi-cuadrado=6,429 g.l. $=2$ sig. $=0,040$ ). 
Figura 3. Tiempo dedicado a las actividades que realiza en la red

\section{Tiempo dedicado a las actividades en en la red (horas)}

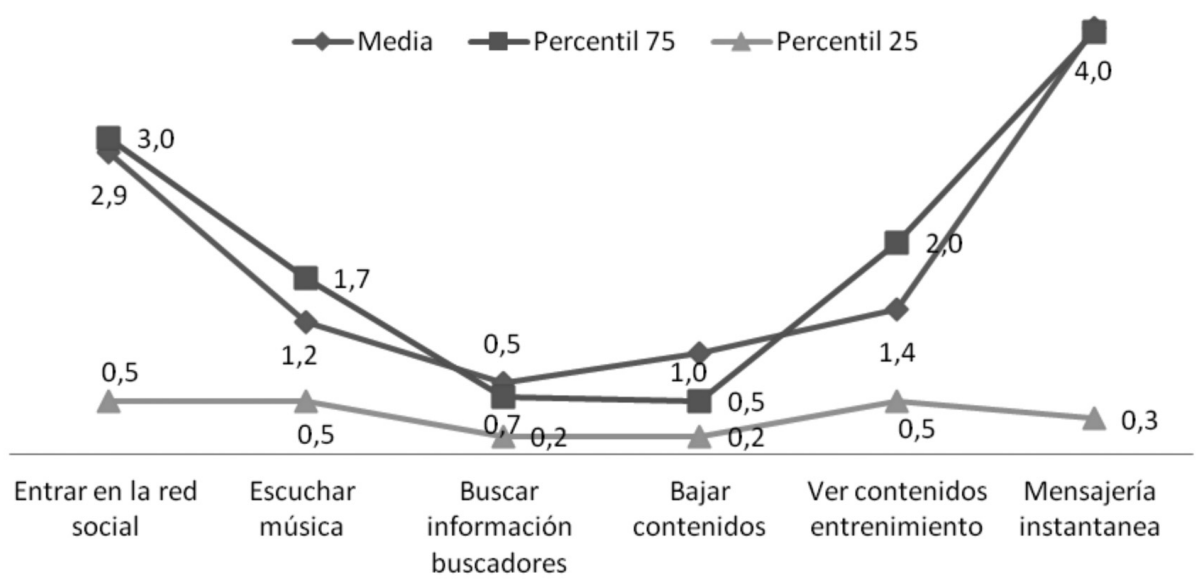

En una jornada habitual se conectan al Tuenti varias veces a lo largo del día, o bien la aplicación queda permanentemente abierta las 24 horas, una nueva posibilidad entre los jóvenes y adolescentes facilitada por los smartphone. Los más jóvenes destacan la accesibilidad de estos dispositivos: "antes veías imposible tener una blackberry y ahora te cambias de compañía y te regalan una" (Grupo 4, adolescentes). Los que la tienen, reconocen que pasan más tiempo conectados y que se lo restan a otras actividades, principalmente al estudio: "a veces voy a hacer los deberes y cuando me doy cuenta ha pasado un ahora y estoy conectada" (Grupo 4, adolescentes). Aunque durante el día chequean varias veces su cuenta, la participación más activa de la red se concentra por la tarde y por la noche: "Es cuando sabes que todo el mundo está conectado y la actividad puede durar hasta las dos o las tres de la madrugada” (Grupo 1, jóvenes).

Reconocen que la posibilidad de estar conectados gracias a estos soportes introduce una paradoja. Por un lado, están permanentemente en contacto, pero por otro se sienten más aislados, y ponen un ejemplo muy ilustrativo:

“cuando estás en un grupo y ves a uno con la blackberry, están todos con la blackberry (...) luego llegas a casa y te pones a hablar con esa persona que tenías delante hace unos minutos por la maquinita" (Grupo 5, adolescentes)

Es decir, que cuando están reunidos en grupo, cara a cara, ven como la utilización de estos dispositivos móviles interfiere en sus relaciones personales. Cada uno de ellos está aislado de ese grupo, pero conectado con otras personas a través de 
la Red, y es más tarde, cuando están en casa, cuando conectan con los amigos con los que acaban de estar en persona y con los que apenas han hablado, pero ahora lo hacen a través del móvil. Reconocen que ellos utilizan la blackberry constantemente, pero dicen que les molesta que los demás lo hagan cuando están reunidos en grupo.

El tiempo dedicado a estar conectado en la red social obtiene en la encuesta un promedio de unas 2 horas y 51 minutos ${ }^{11}$. Ante la posibilidad de que el patrón de consumo se relacione con la edad, se ha llevado a cabo un ANOVA intersujetos para la edad y para el nivel de educación, siendo significativo el efecto del curso ( $F=5,508$; sig. =,005; eta=,062). Los estudiantes de $2^{\circ}$ de Bachillerato muestran mayor moderación en el tiempo dedicado a la conexión de las redes con una media de 1 hora y 54 minutos, frente a las 3 horas de los alumnos de $4^{\circ}$ de la ESO o las 5 horas y 42 minutos del primer curso de bachillerato.

\section{La lista de contactos y el perfil personal}

Uno de los pilares en los que se asientan las plataformas es su potencial de comunicación en red. La conexión en forma de nodos de la red hace que a medida que vas agregando contactos, puedes llegar a los amigos de tus amigos, lo que amplía exponencialmente las posibilidades de contacto y de interacción. En un primer momento, su inicio en el programa les lleva a activar un gran número de contactos. Pueden tener activados entre 300 y 400, alcanzando casi el máximo que les permite la aplicación. Cuando preguntamos por las personas con las que mantienen una relación fluida el número se limita a unas 15 o 20 aproximadamente. Aquí están muy diferenciadas las categorías de amigos íntimos, amigos y conocidos. Con los amigos íntimos tienen una relación muy intensa, casi a diario; con el resto de amigos y familiares mantienen un contacto más esporádico, la frecuencia suele ser una vez a la semana o cada dos semanas. En la categoría de conocidos la actividad es escasa, en un momento determinado se han agregado por alguna coincidencia, pero si la relación no se ha consolidado los contactos decaen y la relación se limita, en el mejor de los casos, a curiosear en su perfil.

"Tienes un listado de amigos de 200 e igual solo hablas con cuatro; yo por ejemplo tengo 380 contactos en Tuenti, pero hablar, hablar con 20 y felicito a unas 40 personas. Incluso a veces (el Tuenti) te avisa del cumpleaños de alguien te metes y dices ieste quién es?"(Grupo 2, jóvenes). Otro participante afirma: “Yo tengo menos, 280 contactos, pero los conozco a todos y el contacto más diario con unas 15 personas" (Grupo 2, jóvenes).

Todos coinciden en que no incorporan a desconocidos en su lista de contactos. A la mayoría los conocen personalmente, pero ello no implica que tengan una relación de confianza, como se ilustra a continuación por uno de los participantes del grupo de jóvenes: "igual tienes a gente que has visto una vez, gente que has conocido en un campamento, una fiesta o incluso compañeros de clase, que son compañeros, pero no son amigos”(Grupo 6, adolescentes). El anonimato de la red permite

11 Tomando como referencia la mediana la mitad de los jóvenes permanece una hora conectado a su red social, para el 25\% de los jóvenes esta actividad se extiende entre 1 y 3 horas, y para otro $25 \%$ supera las tres horas. 
tener una relación más fluida y desinhibida con personas con las que el contacto presencial es muy escaso. La red favorece las interacciones, sin los inconvenientes de la comunicación cara a cara.

Establecen una clara diferencia entre la esfera pública y la privada. Con los que son amigos más íntimos la interacción se mantiene en la esfera privada "le mandas un privado", y los contenidos, comentarios, enlaces, etc. del perfil se hacen de cara a la galería, es decir, la esfera pública. El perfil representa la identidad virtual de cada usuario, la carta de presentación para aquellos contactos que sientan curiosidad. Las fotos, los comentarios y los contenidos que están visibles lanzan una imagen de cada persona. Es fácil perderse en la maraña mediática curioseando perfiles, una actividad que según señalaron los participantes ocupa bastante tiempo, pero que es considerada como una actividad que no es prioritaria, se deja para momentos en los que tienen tiempo. La expresión más utilizada es "si me aburro, entonces me pongo a mirar perfiles...” (Grupo 3, jóvenes), "Permite descubrir cosas interesantes, imágenes, vídeos o un tipo de música que le gusta a esta persona y que de otros modo no hubieras encontrado nunca" (Grupo 3, jóvenes). En definitiva, aporta nuevas referencias y puntos de conexión con la red; al tiempo que permite descubrir el tipo de identidad de la persona, por el tipo de comentarios que hace, las fotos que tienen colgadas, etc. Te permite categorizarla "saber de qué pasta está hecha" (Grupo 2, jóvenes).

Otra parte de la actividad dentro de la plataforma se concentra en torno a las fotos y los eventos. Subir fotos y etiquetarlas (añadiendo el nombre de las personas que aparecen) ocupa bastante tiempo. De nuevo en este aspecto se produce una tendencia hacia la racionalización de la actividad. El número medio de fotos puede estar en torno a las 1.000 o 1.200. Al principio se suben muchas, luego va bajando la intensidad. En torno a las fotos están los comentarios y una gran fuente de información de lo que ha sido la noche de fiesta, la quedada o cualquier otro evento (real). "Si sales en una foto, rápidamente vas a verlo, o si sale alguien en el que estés interesada (comentario femenino), y lo normal es que añadas algún comentario" (Grupo 1, jóvenes). Son conscientes que las fotos también pueden convertirse en una amenaza a su privacidad, "a veces alguien cuelga una foto donde tu apareces que no te gusta mucho, al etiquetarte automáticamente llega a todos tus contactos, solo si la persona es de confianza le puedes decir que quite la foto” (Grupo 1, jóvenes). Un riesgo del que son cada vez más conscientes, y que no les resulta muy fácil controlar.

Eventos: tejido social y una amenaza a la privacidad

La actividad se concentra también alrededor a los eventos. Anunciar en la red una actividad que va a tener lugar es la forma de convocar a los contactos de la red, que pueden abrir el evento, comentarlo o dejar constancia de si irán, aunque muchas de las afirmaciones de asistiré se quedan sólo en el plano virtual (es decir, no se llega a asistir al evento). Sin lugar a duda, los eventos alimentan el tejido social de la red, pueden ser de bastante utilidad para hacer un trabajo de clase con los compañeros, compartir apuntes o realizar una práctica, según reconocen. Otros muchos eventos proceden de propuestas de personas que no están en el círculo más cercano. Los más escépticos no confían en este tipo de acciones y ni 
siquiera las abren, salvo que parezcan interesantes por el título; toman con cautela las invitaciones de estas características puesto que abren la puerta de su perfil a desconocidos. "si comentas en un evento ya sabes a lo que te expones" (Grupo 2, jóvenes). Haciendo referencia a que su perfil es visible a todas las personas que también aceptaron ese evento.

El incremento de la frecuencia de uso puede llegar a saturar y disminuir su eficacia "si abres el Tuenti y te encuentras con 20 eventos ni los miras, le das a descartar salvo que haya algo que veas que te interesa" (Grupo 3, jóvenes). Aparecen muchos eventos "tonterías" simplistas del tipo "lee esto y pásalo a 100 personas y si no mañana nadie te querrá" (Grupo 2, jóvenes)". Este tipo de mensajes también les llegaban antes por el Messenger. Solo los más activos crean eventos o suben otro tipo de contenidos a su perfil, como vídeos o imágenes. Este tipo de acciones es mucho menos frecuente, sobre todo entre los adolescentes, que se consideran "pequeños todavía" (Grupo 3, adolescentes) para generar contenidos".

\subsection{Percepción de riesgo en el medio interactivo y los mecanismos de protección}

Internet es un ámbito que ofrece grandes posibilidades. Ya hemos visto como los jóvenes y los adolescentes destacan de la Red las posibilidades que les ofrece para comunicarse, para buscar información y para el entretenimiento, sin embargo, el medio interactivo es también una fuente de peligros. La red europea de investigación EU Kids Online (2009) clasificó los riesgos derivados de las actividades de los menores en términos de riesgos de contenido (en los que el niño es receptor de contenidos violentos o agresivos, pornográficos, racistas o que inciten al odio y de marketing encubierto), riesgos de contacto (en los que el niño participa de algún modo, aunque sea involuntario, en situaciones de acoso, grooming, persuasión ideológica y uso indebido de los datos personales) y riesgos de conducta (donde el niño es actor de bullying, acoso sexual, contenido generado por usuario potencialmente peligroso y juego, violación de los derechos de autor). El estudio de la Universidad del País Vasco realizado dentro de esta red internacional de investigación determina que el grado de incidencia de la mayoría de los riesgos de Internet que pueden afectar a niños y niñas es menor en España que en la media de los países europeos, pero igualmente preocupante. El 11\% de los menores (9-16 años) españoles afirma haber accedido a contenidos sexuales en Internet, el $9 \%$ ha recibido mensajes de tipo sexual, alrededor de un $5 \%$ de los menores encuestados afirmaron haber sido víctimas de ciberbullying y el $21 \%$ afirmaron haber incluido entre sus contactos de Internet a personas que no conocían cara a cara, por poner algunos ejemplos de los resultados de la macroencuesta (Garmendia, M. et al., 2011).

En nuestra investigación hemos observado grandes diferencias en cuanto a la percepción del riesgo en la red entre los adolescentes. De forma espontánea hay algunos chicos y chicas que reconocen tener muy presente los riesgos que implica Internet y de forma especial las redes sociales, mientras que otros reconocen que las utilizan de forma intuitiva e impulsiva y que no se habían parado a pensar en ese posible riesgo. Uno de los peligros de los que hablan es la dificultad para eliminar la información que has subido a la Red. Comentan algunos casos que conocen personalmente y a 
través de los medios de comunicación: "cuando tú te haces una cuenta y luego la quitas, no te la quitan, siguen quedándose las fotos y todo" (Grupo 4, adolescentes) e incluso hay quien conoce casos en los que "los familiares de una persona fallecida que tenía cuenta en una red social luego tiene problemas para eliminar esa cuenta y la información” (Grupo 5, adolescentes).

Otro de los peligros de la Red es que bajo el anonimato es muy difícil verificar la información y la identidad de las personas: "yo puedo decir que soy un tío y soy una tía", "no están en un cara a cara, estás con un ordenador y no sabes... probablemente te están contando una cosa que no te contarían a la cara, o te están diciendo mentiras. En las relaciones humanas tenemos la voz, los gestos, si hablamos por Tuenti tenemos muchas menos pruebas para saber si lo que están diciendo es verdad" (Grupo 4, adolescentes). En esta línea también se manifiesta otra chica del grupo: "en las redes sociales, como se lee la respuesta, no sabes en qué tono lo está diciendo y yo tuve una pelea por eso" (Grupo 4, adolescentes).

Todos coinciden en que hay que tener cuidado con las fotos que se suben, porque es muy difícil eliminarlas de la Red y pueden ser utilizadas para muchos fines. "Pueden vender las fotos que subes o las pueden utilizar para obtener información para robarte (...) si tú vas en un barco, saben que tienes dinero (...) o para secuestrar a un niño, porque pueden obtener información a través de la Red" (Grupo 5, adolescentes). A pesar de ello, no todos tienen esto en cuenta a la hora de subir las fotos y los comentarios, aunque en el caso de los comentarios también depende de quién los pueda leer: "tengo más cuidado si es una foto en la que hay muchas gente y que la puede ver mucha gente y no si es una foto en la que estoy con una amiga íntima y solo la puede ver ella" (Grupo 5, adolescentes).

A lo largo del discurso son frecuentes las referencias a cuestiones que escapan de su control y que se pueden convertir en amenazas para su privacidad: interactuar con desconocidos, subir fotos, participar en eventos, la publicidad engañosa han surgido de forma espontánea en el discurso. Podríamos afirmar que todos los participantes son conscientes de los riesgos, pero no todos actúan del mismo modo para tratar de evitarlos. No obstante, la responsabilidad recae directamente sobre ellos. Los padres consideran que sus hijos a estas edades son maduros, responsables y capaces de autorregularse por lo que no ejercen ningún tipo de control sobre el tiempo ni sobre el uso y los contenidos a los que acceden. Sus propias experiencias son la principal fuente hacia esa regulación, y no siempre cuentan con las destrezas o habilidades sobre el medio interactivo para evitarlos.

\section{Conclusiones}

El discurso sobre el uso de Internet gira en torno a tres grandes ejes: la comunicación, el entretenimiento y la información, por orden de importancia y tiempo de dedicación. Si hubiera que priorizar entre los tres, Internet es un medio ante todo de comunicación impulsado claramente por las redes sociales. 
El auge de los contenidos audiovisuales y su accesibilidad en la red hace que compita con los medios tradicionales para el consumo. Internet ofrece un abanico muy amplio de contenidos, muchas más posibilidades de elección que favorece un consumo de contenidos a la carta. Otra faceta del entretenimiento viene a través de la música que cobra especial relevancia en la etapa juvenil y suele acompañarlos en múltiples situaciones al compatibilizarse fácilmente con otras actividades (estudiar, buscar información, estar en la red social, etc.).

La comparación entre las características de la migración digital de los jóvenes y los adolescentes nos lleva a concluir que hay más coincidencias que diferencias. Entre estas últimas cabría destacar que para los adolescentes, a diferencia de lo que sucede entre los jóvenes, Internet todavía no ha sustituido al aparato de televisión para el consumo de productos audiovisuales. Utilizan la Red para ver películas y algunas series, sobre todo extranjeras, pero prefieren la televisión para seguir sus programas preferidos. El consumo de contenidos audiovisuales está presente en todo el colectivo y prefieren ver los contenidos directamente en streaming, el tiempo dedicado al visionado es mayor que el tiempo dedicado a las descargas. En ambos casos se está produciendo una evolución al visionado directo de los contenidos en la red, frente a la tendencia de bajar contenidos, tendencia que está más consolidada entre los adolescentes. Para el colectivo todo está disponible en la red y accesible en cualquier momento, solo tiene sentido bajarse contenidos para tenerlos disponibles en otros dispositivos.

Los juegos también tienen su espacio en el entretenimiento, los participantes conocen los riesgos asociados a dejarse llevar por las ofertas lúdicas de la red. El consumo obedece a un patrón que en su inicio tiene mayor intensidad, asociada la novedad y cuyo interés decae con el paso del tiempo y finalmente se abandona. De hecho son los adolescentes (entre 14 y 15 años) los que más tiempo dedican a los juegos en la red. Ambos colectivos son conscientes de que este proceso es adictivo y tratan de desarrollar mecanismos de resistencia para evitar que absorba el tiempo de forma obsesiva.

Por otra parte, Internet constituye una fuente de información referencial para este colectivo. El uso de Internet como fuente de documentación contrastada está asociado a actividades y tareas relacionadas con los estudios, desde donde se incentiva su uso. La wikipedia y el wordreference se han convertido en sitios de referencia, al igual que otras páginas, como el rincón del vago, que gozan igualmente de popularidad a pesar de no tener ningún tipo de fiabilidad sus contenidos. Aunque puntualmente emerge en el discurso, es importante incorporar estrategias de búsqueda en la Red desde los centros educativos.

Las redes sociales son, con diferencia, el aspecto más importante que ofrece Internet a estos jóvenes y adolescentes y no se han observado diferencias significativas en cuanto a su uso. La herramienta cristaliza su necesidad de interacción, posibilita construir su propia imagen personal y expresar así su identidad, al margen del mundo de los adultos. La versatilidad, creatividad y flexibilidad de las aplicaciones les proporciona un espacio abierto para la exploración, en constate renovación y cargado de novedades. Este anhelo constante de saber qué está pasando en la red, lleva en sus versiones más extremas a una dependencia para estar continuamente conectado al medio virtual en detrimento de la conexión con su propia realidad. 
Una de las claves del éxito de las redes sociales es la doble vertiente público y privado, esto es, por una parte es un lugar de encuentro con los amigos más íntimos con los que se tiene una relación muy cercana e intensa, desplazando el posicionamiento de otras plataformas muy consolidadas como el Messenger o el sistema de mensajes SMS a través del teléfono móvil. Al mismo tiempo, les permite mantener el contacto con la red de amigos, saber que está pasando y estrechar lazos a través de la actividad vinculada como colgar fotos, los comentarios, subir contenidos, etc. sin los inconvenientes de la interacción cara a cara; al mismo tiempo, la vertiente más pública es una ventana abierta para establecer nuevos contactos con las personas que tienen algún punto de conexión, a través de la comunicación en forma de nodos.

Se observa una tendencia hacia la racionalización del uso de Internet que se pone de manifiesto en el comportamiento dentro de la red social. Una selección más exhaustiva a la hora de agregar amigos (contactos), disminución del volcado de fotografías en el perfil y mayor precaución hacia el tipo de fotos que sube por el mal uso que se pueda realizar posteriormente. En las actividades de la red, manifestadas a través de los eventos, también se aprecia un uso más moderado, puesto que un uso desmedido e indiscriminado acaba por saturar el espacio para la interacción. Este aspecto también se ha visto contrastado estadísticamente en varias actividades de la encuesta cuya práctica se modera entre los alumnos del último curso de bachillerato. El tiempo de conexión en la red social, el visionado de contenidos de entretenimiento o el tiempo dedicado a los juegos en la red son algunos ejemplos de esta tendencia que posiblemente se pueda abordar en próximos trabajos.

Este colectivo tiene una imagen bastante negativa de la publicidad presente en la red. Una percepción que se ha generado por la existencia de reclamos publicitarios que juegan con la honestidad del mensaje, muy abundantes en las páginas de descarga de contenidos audiovisuales gratuitos. Son conocedores de otro tipo de acciones utilizadas por los anunciantes, hacia las que muestran mucha mayor receptividad, pues lejos del componente de rechazo se consideran como ocasiones para el entretenimiento. Hay una predisposición positiva hacia las acciones procedentes de las marcas más prestigiosas, que cada vez tienen más presencia en otros espacios, como en las redes sociales. No obstante, no se mencionan entre los riesgos percibidos el uso mercantilista que tienen las aplicaciones gratuitas, particularmente las redes sociales.

Se han observado grandes diferencias en cuanto a la percepción del riesgo en la red pero no en función de la edad, puesto que hay jóvenes y adolescentes muy conscientes de los peligros y otros que no se lo habían planteado. No hay implicación de los padres en las actividades de sus hijos en Internet, lo que puede resultar lógico en el caso de los jóvenes, pero no tanto en el de los adolescentes. En este sentido sería interesante indagar el efecto de otras variables que puedan influir en la percepción de riesgo y en el comportamiento en la red.

Por último, cabe reseñar las limitaciones del trabajo. A pesar de que el estudio describe los discursos de los jóvenes y adolescentes en la red, nos enfrentamos a un entorno muy cambiante y con una audiencia que se adapta muy rápidamente a los cambios. Por ello, a pesar de que los resultados son bastante útiles para comprender cómo viven los menores el fenómeno global de internet, la investigación sobre este tema debe ser constante. Por otra parte, el estudio cuantitativo 
tiene un tamaño muestral limitado, debido a las restricciones presupuestarias, por ello, los resultados deben ser tomados con cautela a la hora de generalizarlo al resto de la población de menores residentes en España.

\section{Referencias bibliográficas}

AIMC (2012): "Resumen general de resultados EGM. Octubre de 2011 a Mayo de 2012". Disponible en: http://www.aimc.es/Datos-EGM-Resumen-General-.html [Consultado el 30 de julio de 2012]

Arrizabalaga Crespo, C., Aierbe Barandiaran, A. y Medrano Samaniego C. (2010): “Uso de Internet y mediación parental en adolescentes hiperactivos”, Revista Latina de Comunicación Social, 65, pp. 561-572.

Bringué, J.; Sádaba, C. (2009): Nacidos digitales: una generación frente a las pantallas. Madrid: Rialp.

Bringué, J.; Sádaba, C. (2011): Menores y redes sociales. Foro generaciones interactivas. Madrid: Fundación Telefónica.

CONECTA (2009): Generación N. D. Nacidos Digitales. Disponible en: http://www.congresosfactoria.com/gesconet/uploads/Documentacion/24/Generaci\%C3\%B3n\%20Nacido\%20Digital\%20-\%20D.\%20Miguel\%20Mu\%C3\%B1oz.pdf [Consultado el 10 de mayo de 2010]

EIAA (2010): European Media Landscape Report. Disponible en: http://www.eiaa.net/Ftp/casestudiesppt/EIAA_European_Media_Landscape_Report_SUMMARY.pdf [Consultado el 10 de junio de 2011]

Elzo, J. (dir.) (2009): La Transmisión de Valores a Menores. Informe Extraordinario de la institución del Ararteko, Victoria.

Eurobaromenter 72.5 (2010): E-Communications Household Survey. Disponible en: http://ec.europa.eu/information_society/policy/ecomm/doc/library/ext_studies/household_10/report_en.pdf [Consultado el 16 de junio de 2011]

Frutos Torres, B. y Sánchez Valle, M. (2011): “Aproximación empírica a la percepción de la publicidad on display en Internet”, Sphera Pública. Revista de Ciencias Sociales y de la Comunicación, nº 11, pp. 217-235.

Fundación Pfizer (2009): La Juventud y las Redes Sociales en Internet. Disponible en: http://www.fundacionpfizer.org/ docs/pdf/Foro_Debate/INFORME_FINAL_Encuesta_Juventud_y_Redes_Sociales.pdf [Consultado el 16 de junio de 2011]

Garitaonandia, C.; Garmendia, M. (2009): Cómo usan Internet los jóvenes: hábitos, riesgos y control parental. Universidad del País Vasco/Euskal Herriko Unibertsitatea, Bilbao: EU Kids Online.

Garmendia, M.; Garitaonandia, C.; Martínez, G.; Casado, M. A. (2011): Riesgos y seguridad en internet: Los menores españoles en el contexto europeo. Universidad del País Vasco/Euskal Herriko Unibertsitatea, Bilbao: EU Kids Online.

Hasebrink, U.; Livingstone, S.; Haddon, L. (2008): Comparing children's online opportunities and risks across Europe: Crossnational comparisons for EU Kids Online, London: EU Kids Online. Disponible en http: www.eukidsonline.net. [Consultado el 7 de enero de 2010] 
INE (2011): Encuesta sobre el Equipamiento del Hogar. Disponible en: http: http:/ /www.ine.es/ [Consultado el 18 de junio de 2011]

Livingstone, S. (2008): “Taking risky opportunities in youthful content creation: teenagers' use of social networking sites for intimacy, privacy and self-expression”, New Media \& Society, 10(3), pp. 393-411.

Livingstone, S. (2009): “On the mediation everything”, ICA Presidential address Journal of Communication, 59, pp. 1-18.

Livingstone, S. y Haddon, L. (2009): Kids Online: Opportunities and Risks for Children. Bristol: The Policy.

Livingstone, S.; Helsper, E.J.: "Balancing opportunities and risks in teenagers' use of the internet: The role of online skills and family context”, New Media \& Society, 11(6).

Livingstone, S. (2010): “Youthful participation: what have we learned, what shall we ask next?", First Annual Digital Media and Learning Conference: Diversifying Participation, 18-20 February 2010, University of California, San Diego, La Jolla, California. (Unpublished). Disponible en: http://eprints.lse.ac.uk/27219/. Consultado 10 de junio de 2011.

Monge Benito, S.; Olabarri Fernández, M. E. (2011): "Los alumnos de la UPV/EHU frente a Tuenti y Facebook: usos y percepciones", Revista Latina de Comunicación Social, 66, pp. 79-100. Disponible en: http://www.revistalatinacs.org/ 11/art/925_UPV/04_Monge.html [Consultado el 2 de Marzo de 2011]

ONTSI (2012): La Sociedad en Red. Informe Anual de la Sociedad de la Información en España 2012. Disponible en http://www.ontsi.red.es/ontsi/sites/default/files/informe_anual_2012_v10_ultima.pdf [Consultado el 3 de septiembre de 2012]

Rubio Gil, A. (2008): “Adolescentes y jóvenes en la red: factores de oportunidad". Disponible en http:/ /www.injuve.mtas.es/injuve/contenidos.item.action?id=1724774781\&menuId=572069434 [Consultado el 18 de noviembre de 2009]

Rubio Gil, A. (dir.) (2009): Adolescentes y jóvenes en la Red: Factores de oportunidad: Madrid: Injuve.

Sánchez Burón, A.; Fernández Martín, M. P. (2010): Informe Generación 2.0. Hábitos de los adolescentes en el uso de las redes sociales. Estudio comparativo entre Comunidades Autónomas. Madrid: Universidad Camilo José Cela. Disponible en: http://estaticos.elmundo.es/documentos/2010/07/06/Generacion20.pdf [Consultado en 10 de junio de 2011]

Sánchez Valle, M. y Frutos Torres, B. (2010): "Estrategias de la publicidad interactiva dirigidas al público infantil y adolescente”, II Congreso AE-IC Comunicación y desarrollo en la era digital, Málaga.

The Cocktail Analysis (2012): Observatorio de redes sociales, IV Oleada. Disponible en: http://www.slideshare.net/TCAnalysis/4-oleada-observatorio-de-redes-sociales [Consultado en 31 de julio de 2012] 